\title{
Enseñando potenciación, radicación y logaritmación a partir de los bloques de dienes, bloques multibase y el método de splitting ${ }^{1}$
}

Teaching empowerment, establishment and logaritmación from dienes blocks, multibase blocks and method of splitting

Ensino empowerment, estabelecimento e logaritmación dienos

a partir de blocos, blocos MultiBase e método de divisão

Recibido: mayo de 2013

Aprobado: agosto de 2013
Leidy Viviana Pantano Mogollón ${ }^{2}$

Duvan Ferney González Alfonso ${ }^{3}$

\section{Resumen}

Esta experiencia en el aula es una muestra del trabajo realizado con grado quinto de la Institución Educativa Distrital OEA donde se mostrarán los referentes teóricos utilizados para plantear y diseñar las actividades que fueron aplicadas en el transcurso del semestre, con el fin de acercarse a la noción de potenciación, radicación y logaritmación utilizando como herramientas los bloques de Dienes, multibase, el método splitting y las regletas de Cusinaire además se mostrarán algunos logros obtenidos con estos estudiantes para reflexionar finalmente sobre la experiencia como docentes y la importancia de la planeación y el diseño.

Palabras clave: Matemáticas escolares; números; estructuras numéricas; números enteros; teoría de situaciones didácticas; enseñanza; planificación del profesor; metodología de enseñanza.

\begin{abstract}
Esta experiencia en el aula es una muestra del trabajo realizado con grado quinto de la Institución Educativa Distrital OEA donde se mostrarán los referentes teóricos utilizados para plantear y diseñar las actividades que fueron aplicadas en el transcurso del semestre, con el fin de acercarse a la noción de potenciación, radicación y logaritmación utilizando como herramientas los bloques de Dienes, multibase, el método splitting y las regletas de Cusinaire además se mostrarán algunos logros obtenidos con estos estudiantes para reflexionar finalmente sobre la experiencia como docentes y la importancia de la planeación y el diseño.
\end{abstract}

Keywords: Matemáticas escolares; números; estructuras numéricas; números enteros; teoría de situaciones didácticas; enseñanza; planificación del profesor; metodología de enseñanza.

1 Artículo de Investigación.

2 Universidad Distrital Francisco José de Caldas. Bogotá, Colombia. Contacto: lvpmogollon@gmail.com

3 Universidad Distrital Francisco José de Caldas. Bogotá, Colombia. Contacto: duducorreo@gmail.com 


\section{Resumo}

Esta experiencia en el aula es una muestra del trabajo realizado con grado quinto de la Institución Educativa Distrital OEA donde se mostrarán los referentes teóricos utilizados para plantear y diseñar las actividades que fueron aplicadas en el transcurso del semestre, con el fin de acercarse a la noción de potenciación, radicación y logaritmación utilizando como herramientas los bloques de Dienes, multibase, el método splitting y las regletas de Cusinaire además se mostrarán algunos logros obtenidos con estos estudiantes para reflexionar finalmente sobre la experiencia como docentes y la importancia de la planeación y el diseño.

Palavras-chave: Matemáticas escolares; números; estructuras numéricas; números enteros; teoría de situaciones didácticas; enseñanza; planificación del profesor; metodología de enseñanza.

\section{Contextualización}

La innovación en métodos de enseñanza ha venido jugando un papel importante en la forma en que los estudiantes ven la matemática, ya que estos nuevos métodos de enseñanza involucran factores con los cuales los estudiantes pueden interactuar y así lograr una interiorización de los conceptos a enseñar; es por ello que los autores de esta ponencia en el segundo semestre del 2012 realizando su segunda práctica consideraron importante incluir el método de splitting y los bloques de Dienes para la enseñanza de la potenciación, radicación y logaritmación en grado quinto del la I.E.D OEA. Para ello se realiza el diseño y la planeación en concordancia a lo estipulado por el grupo DECA (1992), el cual plantea varias etapas que se adaptaron a la temática de la práctica y al curso correspondiente para desarrollar conceptos del pensamiento numérico y sistemas numéricos.

\section{Marco teórico - prácticos básicos}

Para el desarrollo de las actividades fue necesario a referentes teóricos; siguiendo a Cid, Godino \& Batanero (2002) se tienen las siguientes definiciones Potenciación, son productos repetidos, ya que en vez de escribir $3 \times 3 \times 3 \times 3$ escribimos $3^{4}$ y esto quiere decir que tenemos que multiplicar el número 3 por sí mismo 4 veces. En una potencia $\mathrm{c}=\mathrm{a}^{\mathrm{b}}$ se dice que a es la base y b el exponente. Radicación, en general si $\mathrm{c}=\mathrm{a}^{\mathrm{b}}$ entonces $\mathrm{a}={ }^{\mathrm{b}} \sqrt{\mathrm{c}} \mathrm{c}$. La raíz puede considerarse como operación inversa de la potencia que nos permite encontrar la base, conocida la potencia y el exponente, Logaritmación, puede considerarse como operación inversa de la potencia que nos permite encontrar un exponente conocida una potencia y su base $\log _{a} c=b$. Un ejemplo que menciona Godino de cómo se ven reflejados estos tres conceptos en un ejercicio es: en la igualdad $5^{3}=125$ decimos que 125 es el cubo de 5 pero también podemos decir que 5 es la raíz cúbica de 125 y que 3 es el logaritmo en base 5 de $125.5^{3}=$ $125, \sqrt[3]{125}=5, \log _{5} 125=3$

La potenciación, radicación y logaritmación a menudo son conceptos difíciles de aprender e interiorizar, puesto que los estudiantes no pueden hacer redes de generalizaciones al respecto, para ello se propone que estos conocimientos sean enseñados por medio del método de splitting que consiste en recrear la unidad a partir de los splits, claro está que los splits deben ser iguales; para Confrey (1994, Pág. 300) un split es un precursor de un concepto más adecuado de razón y proporción y por ende, de razón de cambio multiplicativa y funciones exponenciales y logarítmicas. Un split es una acción de crear partes iguales o copias de un original. El Splitting es una operación primitiva que requiere sólo el reconocimiento del tipo de split y el requerimiento de que las partes sean 
Figura 1.

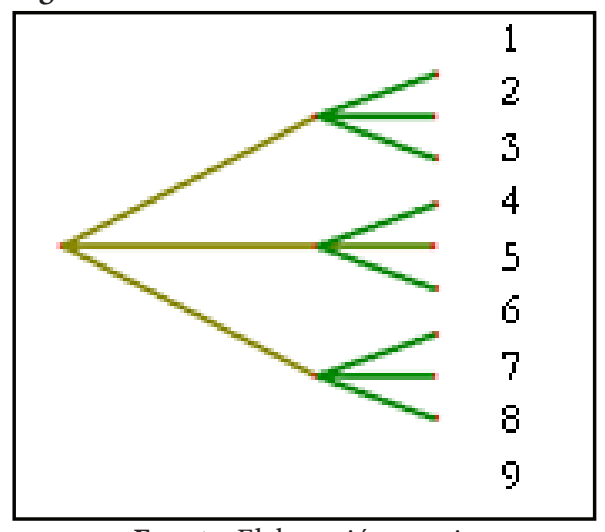

Fuente: Elaboración propia

iguales, por ejemplo para las potencias del tres ver figura 1.

Y teniendo en cuenta lo estipulado en las definiciones de potenciación, radicación y logaritmación dadas anteriormente se puede construir una a partir de la otra, es decir, este método construye las potencias de un número entero; pero a partir de este se puede deducir la radicación y la logaritmación del mismo.

Otras herramientas utilizadas fueron los bloques de Dienes (ver figura 2), donde se construye las potencias del tres y los bloques multibase (ver

Figura 2.

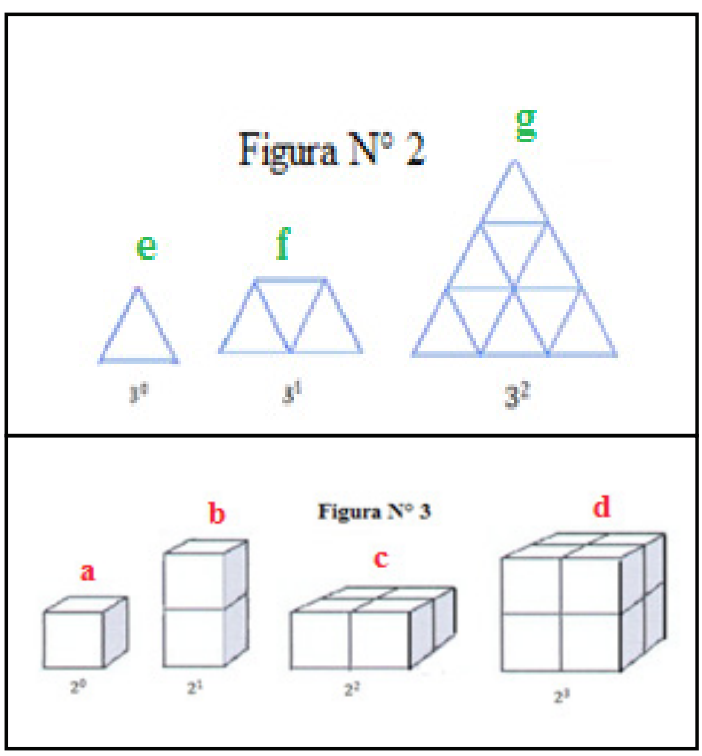

Fuente: Elaboración propia figura 3), donde se construye las potencias del dos por medio de preguntas como se menciona más adelante.

La enseñanza de todos estos conceptos se da a partr de los procesos generales que se estipulan en los Lineamintos curriculares para matemáticas (1998), los cuales son: razonamiento, modelación, comunicación, formulación y ejercitación de procedimientos y formular y resolver problemas, claro está que estos procesos no se dan en una escala determinada sino que van apareciendo a medida que los estudiantes resuelven las actividades. Todas las actividades que se describirán en seguida fueron planeadas y diseñadas teniendo como base lo expuesto por el grupo DECA (1992), ellos plantean tres etapas, donde la primera es la fase de iniciación e introducción, la segunda es la fase reestructuración y profundización, y la última fase es la de evaluación, las cuales se adaptaron en una serie de actividades que mencionaremos más adelante.

\section{Descripción general de la experiencia en el aula, logros y dificultades evidenciadas}

Durante el transcurso de la práctica (experiencia en el aula) se realizaron 9 actividades, las cuales se estructuraron de la siguiente manera: Iniciación e introducción: actividad de reconocimiento, en ella se buscaba reconocer a los estudiantes y determinar algunas características de los mismos, para ello se planteo una actividad donde los estudiantes debían hacerse por parejas, donde uno era el lazarillo y el otro el pintor, el lazarillo debía indicarle al pintor todos los movimientos para poder realizar una determinada obra ya que el pintor era una persona invidente, en esta se evidencio que la mayoría de los estudiantes tenían la facilidad para trabajar en grupo, puesto que se metieron en el papel de lazarillo y pintor trabajando en equipo para lograr buenas obras; pero se presentaron dificultades como el no saber escuchar, ya que algunos grupos no realizaron adecuadamente el trabajo. La segunda actividad fue la prueba diagnóstico donde se evaluaría los conceptos que tenían los estudiantes hasta ese momento, que fueran pre-requisito para interiorizar los conceptos que se iban a enseñar; la prueba tuvo los siguientes temas: aplicación 
de las operaciones básicas y sus propiedades, numeración decimal, romana y binaria, y números decimales y sus operaciones. Allí se evidencio que los estudiantes no podían extraer rápidamente las respuestas de las situaciones problema, según Godino, Batanero \& Font (2003) es debido a que la situación problema no contempla al estudiante como actor, además no tenían aspectos del aula con los que él pudiera palpar la situación en otros caso los estudiantes cometían errores de tipo procedimental y conceptual como por ejemplo al abstraer los elementos del problema (para la multiplicación el multiplicador y el multiplicando y para la división el divisor y el dividendo) por dos razones, cuando nunca se han visto enfrentadas a ellas y por tanto tratan de resolverlas por sumas o restas y cuando no es aprendido ni interiorizado el concepto.

Reestructuración y profundización: en esta fase se trabajo:

La actividad $N^{\circ} 1$ se llamo "Reconociendo mi instrumento de trabajo" en la cual se buscaba que los estudiantes aprendieran a utilizar la regletas de cusinaire, por medio de ellas se pretendía enseñar los temas a trabajar durante la práctica, en esta actividad se les hacía entrega de un juego de regletas, seguido de una breve explicación sobre el recurso y luego se le entrega a los estudiantes una guía con una serie de preguntas que debían responder teniendo en cuenta las regletas, en la actividad se evidencio que para los estudiantes fue difícil ver que las regletas pueden "remplazar" los números como símbolos y tomar su posición para trabajar en colores; esto es comprensible según (Godino et al., 2003) porque para un estudiante siempre será difícil salirse de un contexto conocido para internarse en uno completamente desconocido.

La actividad $N^{\circ} 2$ se llamo construyendo torres de colores la cual consistía en que por medio de las regletas de Cusinaire los estudiantes llegaran al concepto de potenciación, en esta actividad los estudiantes no dedujeron la potenciación puesto que el recurso utilizado (regletas de cusinaire) no les eran familiares ni les llamaba la atención, la actividad presento muchas dificultades entorno a la construcción del objeto matemático, es por ello que se decide cambiar el recurso.

La actividad $N^{\circ} 3$ consistía en, por medio del método de splitting los estudiantes llegaran al concepto de potenciación ya que en la anterior sesión no se logro con las regletas de cusinaire; en esta actividad la mayoría de los estudiantes llegaron al concepto de potenciación dado que la actividad propuesta los encaminaba a conjeturar y validar para así llegar a la construcción del mismo, claro está que algunos estudiantes no lo lograron porque no quisieron desarrollar la actividad, luego los docentes institucionalizaron el concepto.

La actividad $N^{\circ} 4$ consistía en trabajar la radicación a partir del método de splitting y los bloques de Dienes y multibase, por medio de una serie de preguntas donde se tomaba lo construido en la sesión anterior para interiorizar el nuevo concepto; la dificultad más evidente fue la ausencia de estudiantes a la actividad, aunque los estudiantes que asistieron no lograron construir e interiorizar el concepto a través de la actividad, ya que ellos no tomaban lo anterior para darle desarrollo a la actividad, es por ello que se plantea otra actividad con el fin de que los estudiantes logren interiorizar y construir el concepto.

La actividad $N^{\circ} 5$ consistía en trabajar los bloques de Dienes y los bloques multibase en la cual se formulaban una serie de preguntas que les permitían a los estudiantes construir el concepto de radicación lo cual se evidencio al analizar los trabajos realizados por los estudiantes ya que al ver los bloques de Dienes y multibase, sintieron cierta familiaridad, aunque cabe mencionar que al principio de la actividad algunos estudiantes tuvieron un razonamiento pobre como lo menciona Russell, J. (1999); pero estos estudiantes fueron incitados a realizar razonamiento matemático con diferentes preguntas hechas por los docentes, dando como resultado la construcción del concepto.

La actividad $N^{\circ} 6$ consistía en trabajar el concepto de logaritmación por medio del método de splitting y los bloques de dienes y multibase a partir de estas se plantearon una serie de preguntas que los llevaría a dicho concepto, se evidencio que los 
estudiantes lograron construir el conocimiento con más facilidad ya que conocían perfectamente las herramientas trabajadas para dichos conceptos. Como se menciono a lo largo del capítulo se establecieron una serie de preguntas para trabajar los temas como por ejemplo: Figura 1: ¿Qué relación hay entre la cantidad de ramas de cada color?, escribe la cantidad de ramas verdes en términos del 3, haz el nivel que le sigue a las ramas verdes; Figura 2: ¿Qué número debo multiplicar dos veces por sí mismo para obtener el triángulo g?, ¿Qué número elevado a la 1 me da el triángulo $\mathrm{f}$ ? ¿Por qué es ese número?, Figura 3: 2 elevado a la

me da la cantidad de cubos de la figura b. ¿Por qué?, ¿Cómo se llama el número que estoy buscando en los puntos anteriores, Es el mismo número que se halla en la potenciación y en la radicación? Si/no ¿Por qué?, entre otras

Evaluación: se realizó la actividad de cierre, en esta actividad se evaluaron los conocimientos de los estudiantes, pero allí debían aplicarlos en situaciones diferentes a las trabajadas en clase, con el fin de ver si los estudiantes interiorizaron los conceptos; la mayoría pudo aplicar los conceptos en las situaciones planteadas. Con esto se puede concluir que la práctica (experiencia en el aula) fue exitosa en tanto que los estudiantes interiorizaron los conceptos y allí se ve que la innovación de los recursos y modelos de enseñanza dan buenos resultados siempre y cuando estos sean del agrado de los estudiantes.

\section{Reflexión Final}

En nuestra opinión esta experiencia en el aula fue gratificante ya que cambiamos en algo la forma en la que los estudiantes aprenden los conocimientos, si bien aprendieron un concepto lo importante es cambiar o dejar una huella en ellos que les servirá para el futuro. Como docentes tenemos una clara obligación de no solo educar niños en matemáticas o en biología o en cualquier asignatura, sino educarlos en ser personas y miembros activos de una sociedad, que la podrán mejorar y encaminar hacia un mejor futuro.

\section{Referencias}

Confrey, J. (1994). Splitting

Godino, J., Batanero, C., Cid, E. (2002). Sistemas de numeración y su didáctica para maestros.

Godino, J., Batanero, C., Font, V. (2003). Fundamentos de la enseñanza y el aprendizaje de las matemáticas para maestros. Granada

Grupo DECA (1998). Orientaciones para el aprendizaje y elaboración de actividades de aprendizaje y de evaluación. Revista Aula N ${ }^{\circ} 7$

Ministerio De Educación Nacional. (1998). Lineamientos Curriculares De Matemáticas. Bogotá, Colombia.

Russell, S. (1999). Razonamiento matemático en los primeros grados. 